\title{
Layer 3 Throughput Analysis for Advanced ALOHA Protocols
}

\author{
Federico Clazzer \\ German Aerospace Centre (DLR) \\ Institute of Communications and Navigation \\ Oberpfaffenhofen, 82234, Wessling, Germany \\ Email: federico.clazzer@dlr.de
}

\author{
Mario Marchese \\ DITEN - Department of Electrical, Electronic, and \\ Telecommunications Engineering, and Naval Architecture \\ Via Opera Pia 13, 16145, Genova, Italy \\ Email: mario.marchese@ unige.it
}

\begin{abstract}
Random Access (RA) Medium Access (MAC) protocols are simple and effective when the nature of the traffic is impulsive and unpredictable. Recently, evolutions of RA have shown impressive improvements in the throughput, but no investigation on the impact on the higher layers has been carried out, at the best of our knowledge. The objective of the paper is to investigate recently presented RA solutions in terms of layer 3 throughput when generic packet length distribution is considered. The definition of layer 3 throughput is presented for both slotted and non slotted protocols. In the latter case, a comprehensive analytical framework is developed, where different levels of knowledge of the packet distribution are considered. The results show that non slotted protocols that use Forward Error Correction (FEC) schemes are able to outperform slotted protocols under the same conditions. On the other hand, the layer 3 throughput of non slotted protocols is more sensitive to changes in the form of the packet length distribution than in slotted protocols.
\end{abstract}

\section{INTRODUCTION}

Since the introduction of the ALOHA protocol by Abramson in the early 70's [1], many new RA protocols have been developed. RA MAC protocols are particularly attractive for all scenarios where the traffic is unpredictable and random, such as satellite return links and ad hoc networks.

In the recent past, Contention Resolution ALOHA (CRA) [2] and Contention Resolution Diversity Slotted ALOHA (CRDSA) [3], evolutions of respectively ALOHA and Slotted ALOHA [4], [5], have been presented as very promising protocols where the idea is to use multiple identical packets sending per user at the transmitter and a smart collision recovery with Successive Interference Cancellation (SIC) at the receiver ${ }^{1}$. In this way, both protocols can achieve an impressive throughput increase with respect to the original ALOHA and Slotted ALOHA. More recently, a further improvement in the slotted protocols has been achieved with the introduction of Irregular Repetition slotted ALOHA (IRSA) [6], where the number of replicas for each user is drawn from an optimal distribution that maximizes the throughput. Also concerning non slotted protocols, recently presented papers have shown the possibility to boost further the throughput using smart packet recombination [7], [8].

\footnotetext{
${ }^{1}$ More details will be given in the next section.
}

For new RA protocols the investigation of higher layer throughput is a very important and still a missing element in the state-of-the-art. The possibility to exploit the gain in terms of throughput also at higher layers can open to RA protocols new application scenarios where RA was not considered as suitable.

The objective of the paper is to analyse and investigate the layer 3 throughput behavior of framed non slotted and slotted RA protocols, such as CRA and CRDSA, assuming a generic layer 3 packet length distribution $f_{X}(x)$. In other words, we assume that higher layers will generate packets with a given length distribution $f_{X}(x)$ and we will investigate the impact on the layer 3 throughput in consequence of RA actions.

\section{SySTEM MODEL}

We consider a population of $N_{u}$ users sharing the same medium. Each user generates packets of layer 3 where the duration is subject to a generic probability measure $f_{X}(x)^{2}$. The probability measure captures the behavior of the traffic profile followed by the users. We assume that the probability measure $f_{X}(x)$ is equal for all the users and can be either continuous or discrete. The user cannot forward the layer 3 packet to layer 2 until the previous layer 2 packet has been sent. Furthermore, for the investigation carried out in this paper, the protocol is assumed to work in open loop, i.e. no feedback and packet acknowledgment procedure is here considered.

All the layer $2 \mathrm{MAC}$ protocols evaluated in the following are RA, either non slotted or slotted. We consider ALOHA, CRA, Slotted ALOHA (SA) and CRDSA. The analysis performed in section III is independent of the specific protocol and can be applied also to other RA schemes ${ }^{3}$. A framed time structure is assumed for all protocols. In this way, the time is composed by frames of $T_{f}$ [s] duration. Each user can try to transmit only once for each MAC frame and we assume that all users have always packets to be sent. Upon the generation of more than one packet per frame, the users need to store

\footnotetext{
${ }^{2}$ The probability measure $f_{X}(x)$ corresponds to the Probability Density Function (PDF) in case of continous random variable, or corresponds to the Probability Mass Function (PMF) in case of discrete random variable

${ }^{3}$ The words scheme and protocol will be used interchangeably in the following.
} 


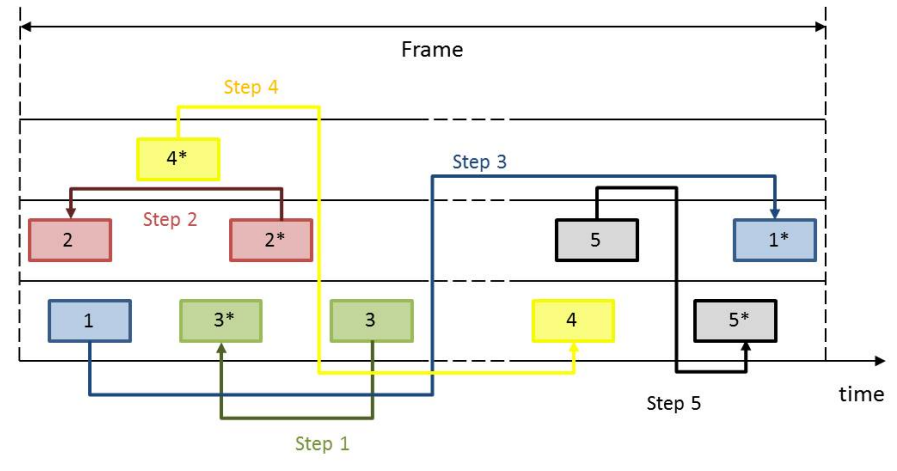

Fig. 1. SIC procedure in CRA. The packets are placed in different levels only for simplify the visualization, but they are assumed to be sent in the same frequency and therefore they are partially overlapping in the frame. The SIC procedure starts from the first packet free from interference which is packet 3 . Once it is successfully decoded its twin replica, packet $3 *$ can be removed from the frame. In this way, packet $2 *$ can be correctly decoded and its twin (packet 2) is also removed from the frame. The SIC procedure proceeds until all the packets can be successfully decoded.

or discard the exceeding packets (depending on the protocol implementation). The physical layer packets are supposed to have a duration of $T_{p}$ [s]. Concerning slotted protocols, the frames are further subdivided in slots of a fixed duration $T_{s}$ [s]. In slotted schemes, the physical layer packet duration has to be equal to the slot duration in order to fit in the time slot, i.e. $T_{p}=T_{s}$, while in non slotted schemes this constraint can be relaxed.

We define the offered traffic load $G$ as the fraction of time occupied by transmissions. In formulas, $G=\frac{N_{u} \cdot T_{p}}{T_{f}}$. In the slotted schemes we can write also $G=N_{u} / M$, where $M=$ $T_{f} / T_{p}=T_{f} / T_{s}$ is the number of slots that constitute each MAC frame.

A brief review of the MAC protocols investigated in the reminder of the paper is carried out in the next two subsections.

\section{A. Non slotted MAC protocols, ALOHA and CRA}

In the ALOHA protocol, users are allowed to try accessing the medium whenever they have packets to send and independently of the activity on the medium. In case of packet collision, all involved packets are lost. As known from literature, ALOHA throughput is quite limited, only 0.18 .

CRA extends the ALOHA protocol employing multi-packet generation at the sender and SIC procedure at the receiver. Before transmitting, CRA (1) replicates the packet $d$ times, (2) selects the instants when to send this $d$ replicas of the packet in the MAC frame and (3) adds a pointer to the replicas location in all the $d$ identical replicas ${ }^{4}$.

At the receiver side, the SIC procedure is employed in the CRA protocol. The SIC procedure starts from the beginning of the frame and looks for a decodable packet. Once found, the

\footnotetext{
${ }^{4}$ The physical offered traffic load of CRA is $d \cdot G$, but the $d$ replicas are identical. Therefore, this quantity does not provide the information of the traffic effectively managed by the protocol, which is instead captured by $G$ solely.
}

packet is (1) decoded, (2) the pointer to the replica locations is extracted and (3) the waveform is reconstructed and removed from the frame locations where the $d$ replicas are placed. This procedure can retrieve some packets collided with the $d$ replicas just deleted and can boost the protocol throughput. The SIC procedure is iterated until either all the packets have been successfully decoded or a maximum number of iterations is reached (see figure 1 for CRA SIC procedure and its caption for a detailed example). In this way, the throughput can be increased to 0.32 (CRA with $d=3$ and no FEC) or even more ( 0.96 with $d=2$ and rate $R=1)$ when FEC is assumed [2].

\section{B. Slotted MAC protocols, SA and CRDSA}

The SA protocol is an evolution of the ALOHA protocol in which the time is divided in slots of equal duration. Users are allowed to access the medium only upon the beginning of the slots and, as in ALOHA, destructive collisions may happen. In SA, if a packet is generated in the interval between two consecutive slots, the packet can be sent only when the subsequent slot starts. The SA throughput is quite limited in absolute terms, only 0.36, but it doubles the one of ALOHA.

CRDSA is an extension of the SA where $d$ identical replicas are sent per frame by the users through the medium and SIC procedure is employed at the receiver in order to resolve collisions ${ }^{5}$. This protocol is able to reach a maximum throughput of 0.52 with $d=2$ and 0.68 with $d=3$.

\section{L3 THROUGHPUT ANALYSIS}

The throughput $S$ is one of the mostly used performance metrics for RA protocols and it is defined as the successful decoding probability $P_{\text {succ }}$ multiplied by the offered traffic load $G$

$$
S=G \cdot P_{\text {succ }}\left(G, f_{X}(x)\right) .
$$

In general, the successful decoding probability depends on the offered traffic load $G$ and on the packet length distribution $f_{X}(x)$. For simplicity of notation these dependencies are neglected in the following computations.

\section{A. Non slotted RA MAC protocols}

When non slotted framed protocols, as ALOHA or CRA are employed, all layer 3 packet durations non exceeding the frame duration can be accommodated in the MAC frame. In this way, no fragmentation of layer 3 packets has to be performed and successful decoding probability of layer 3 packets $P_{s u c c-L 3}$ coincides with the successful decoding probability of layer 2 packets $P_{\text {succ-L2 }}$. Therefore, the throughput $S_{L 3-\text { unslotted }}$ for layer 3 in case of non slotted schemes is expressed as in (1)

$$
\begin{aligned}
S_{L 3-\text { unslotted }} & =G \cdot P_{\text {succ-L3 }}=G \cdot P_{\text {succ-L2 }}= \\
& =S_{L 2-\text { unslotted }} .
\end{aligned}
$$

\footnotetext{
${ }^{5}$ The protocol behavior is very similar to CRA. Please refer to the previous subsection for the detailed transmitter and receiver protocol operations.
} 


\section{B. Slotted RA MAC protocols}

Although users at layer 3 generates packets whose durations are ruled by $f_{X}(x)$ probability measure, slotted schemes cannot accommodate packets with generic duration at MAC layer and fragmentation is needed. When a layer 3 packet has to be fragmented, additional overhead is added to the fragmented layer 2 packets. Two types of overhead are added to the layer 2 packets: (i) fragmentation overhead and (ii) padding overhead. The former is due to the need to have a layer 3 header for each fragment, where the fields to both route the packet in the network and to recompose the packet at the destination are contained. Header fields dedicated to fragmentation must include: original-not-fragmented packet identifier, fragment identifier, and either overall number of fragments or indicator about last/not last fragment. For example, concerning Internet Protocol (IP) layer 3 protocol, the fields identification, offset and flag $\mathrm{M}$ are used, respectively, for the aims listed above. The padding overhead is needed in order to fulfill layer 2 slot duration requirements.

Layer 3 throughput $S_{L 3-\text { slotted }}$ for slotted schemes is:

$$
\begin{array}{r}
S_{L 3-\text { slotted }}=G \cdot P_{\text {succ-L3 } 3} \cdot\left(1-O_{\text {frag }}\right) \cdot\left(1-O_{\text {pad }}\right), \\
\text { with } 0 \leq O_{\text {frag }} \leq 1 \\
\text { with } 0 \leq O_{\text {pad }} \leq 1
\end{array}
$$

where $P_{\text {succ }-L 3}$ is the successful decoding probability of layer 3 packets, $O_{\text {frag }}$ is the fragmentation overhead and $O_{\text {pad }}$ is the padding overhead.

The successful decoding probability of layer 3 packets $P_{\text {succ-L3 }}$ in the slotted schemes is the probability that all the layer 2 packets composing the layer 3 packet are received correctly weighted with the probability that this specific layer 3 duration is selected,

$$
\begin{aligned}
P_{\text {succ }-L 3} & =p_{1} P_{\text {succ }-L 2}+p_{2} P_{\text {succ }-L 2}{ }^{2}+\ldots \\
& +p_{n} P_{\text {succ }-L 2}{ }^{n}+\ldots= \\
& =\sum_{i=1}^{\infty} p_{i} P_{\text {succ-L2 }}{ }^{i} \quad \text { with } \sum_{i=1}^{\infty} p_{i}=1 .
\end{aligned}
$$

1) Example 1, $f_{X}(x)=\frac{1}{2} \delta(x-1)+\frac{1}{2} \delta(x-2)$ : We can suppose for example, that the layer 3 packet duration distribution PMF $f_{X}(x)$ results in $50 \%$ of layer 3 packets with a duration of 1 layer 2 packet and $50 \%$ of layer 3 packets with a duration of 2 layer 2 packets, i.e. $f_{X}(x)=\frac{1}{2} \delta(x-1)+\frac{1}{2} \delta(x-2)$. The layer 3 successful decoding probability for the first packet duration (1 layer 2 packet) is simply $P_{\text {succ }-L 2}$. Actually, we need only that the layer 2 received packet is successfully decoded. While for the second packet duration (2 layer 2 packets) the probability is $P_{\text {succ }-L 2}{ }^{2}$ because two consecutive layer 2 packets must be successfully decoded. In this way, equation (3) becomes for this example

$$
P_{\text {succ-L3 }}=\frac{1}{2} P_{\text {succ-L2 }}+\frac{1}{2} P_{\text {succ }-L 2}{ }^{2} .
$$

Note that $P_{\text {succ }-L 2}$ is in closed form if we consider the SA protocol. In this case $P_{\text {succ }-L 2}=e^{-G}$ assuming an infinite user population [5] and we can further write

$$
P_{\text {succ-L3 }}=\frac{1}{2} e^{-G}+\frac{1}{2} e^{-2 G} .
$$

2) Computation of $p_{i}$ : In general $f_{X}(x)$ can be continuous, i.e. the layer 3 packet durations maybe not constrained to be a multiple of layer 2 packet durations. In these scenarios the computation of $p_{i}$ is of paramount importance to determine the layer 3 successful decoding probability $P_{\text {succ }-L 3}$. The generic $p_{i}$ value is computed as the definite integral of the PDF $f_{X}(x)$ in the interval from the layer 2 duration $i-1$ to the layer 2 duration $i$, where $i=1, \ldots, \infty$ are discrete layer 2 durations ( $i=1$ means 1 layer 2 packet duration),

$$
p_{i}=\int_{i-1}^{i} f_{X}(x) d x \quad \text { for } i=1, \ldots, \infty .
$$

3) Example 2, $f_{X}(x)=e^{-x}$ : As second example, we assume $f_{X}(x)=e^{-x}$, the exponential distribution with mean of 1 layer 2 packet duration. In this example, accordingly to equation (4) $p_{i}$ are

$$
\begin{aligned}
p_{i} & =\int_{i-1}^{i} f_{X}(x) d x=\int_{i-1}^{i} e^{-x} d x=\left[-e^{-x}\right]_{i-1}^{i}= \\
& =\frac{1}{e^{i-1}}-\frac{1}{e^{i}} \quad \text { for } i=1, \ldots, \infty .
\end{aligned}
$$

After the discretization the mean of the exponential distribution will be greater than 1 layer 2 packet duration. This is due to the discretization process which is a ceiling operation.

In general, the expected value of the discretized version of $f_{X}(x)$ called $\mathbb{E}\left[\overline{f_{X}(x)}\right]$ can be written as

$$
\mathbb{E}\left[\overline{f_{X}(x)}\right]=\sum_{i=1}^{\infty} i \cdot p_{i},
$$

where the result is expressed in layer 2 packet duration. The values of $p_{i}$ are computed according to equation (4). In the case of the exponential distribution equation (5) becomes

$$
\mathbb{E}\left[\overline{f_{X}(x)}\right]=\frac{e}{e-1} \cong 1.58
$$

which is greater than 1 layer 2 packet duration, as expected.

4) $f_{X}(x)$ unknown: In some situations $f_{X}(x)$ or its discretized version are not known. This prevents the analytical computation of $p_{i}$, but we would like to be able to analytically evaluate the successful decoding probability of layer 3 packets $P_{\text {succ }-L 3}$ also in these situations. We assume that the expected value of the probability measure's discretized version $\mathbb{E}\left[\overline{f_{X}(x)}\right]$ can be measured at the receiver ${ }^{6}$. We can then approximate the successful decoding probability of layer 3 packets $P_{\text {succ }-L 3}$ as

\footnotetext{
${ }^{6}$ The packets correctly decoded will contain the sequence number needed for layer 3 packet reconstruction and it can be used for numerically evaluate the $\mathbb{E}\left[\overline{f_{X}(x)}\right]$
} 


$$
P_{\text {succ-L3-appr }}=\left(P_{\text {succ-L2 } 2}\right)^{\mathbb{E}\left[\overline{f_{X}(x)}\right]} .
$$

It can be demonstrated that $P_{\text {succ-L3-appr }} \leq P_{\text {succ-L3 }}$ using the Jensen's inequality [9] ${ }^{7}$, i.e. the approximated successful decoding probability of layer 3 packets is a lower bound of the exact successful decoding probability of layer 3 packets. In this way equation (2) can be rewritten as

$$
\begin{array}{r}
S_{L 3-\text { slotted }} \geq G \cdot P_{\text {succ-L3-appr }} \cdot\left(1-O_{\text {frag }}\right) \\
\cdot\left(1-O_{\text {pad }}\right), \text { with } 0 \leq O_{\text {frag }} \leq 1 \\
\text { with } 0 \leq O_{\text {pad }} \leq 1 .
\end{array}
$$

Numerical results of the difference between $P_{\text {succ-L3 }}$ and $P_{\text {succ-L3-appr }}$ in terms of layer 3 throughput are given in the next section.

5) Final remarks: The analytical framework developed in this subsection provides the possibility to evaluate the layer 3 throughput $S_{L 3 \text {-slotted }}$ in case of slotted schemes without the need of numerical simulation of this layer. Two possibilities can be followed

1) $P_{\text {succ }-L 2}$ and $f_{X}(x)$ are provided (see equations (2), (3), (4))

2) $P_{\text {succ }-L 2}$ and $\mathbb{E}\left[\overline{f_{X}(x)}\right]$ are provided (see equations (7), (6))

\section{NUMERICAL RESUlTS}

In this section a comparison between non slotted and slotted MAC protocols will be provided. Two distributions of layer 3 packet duration are considered: equal probability of 1 layer 2 and 2 layer 2 packet durations (see first example in previous section), i.e. $f_{X}(x)=\frac{1}{2} \delta(x-1)+\frac{1}{2} \delta(x-2)$; exponential packet length distribution with mean 1 layer 2 packet duration (see second example in previous section), i.e. $f_{X}(x)=e^{-x}$. Finally, a comparison between the layer 3 throughput of slotted schemes in case of exact successful decoding probability $P_{\text {succ-L3 }}$ and the approximated one $P_{\text {succ-L3-appr }}$ will be also provided.

\section{A. Non slotted and slotted RA MAC protocols layer 3 through- put comparison}

The simulations performed show the layer 3 throughput $S_{L 3}$, which is the generic notation for $S_{L 3-\text { slotted }}$ and $S_{L 3-\text { unslotted }}$ presented in the previous sections, averaged over $10^{3}$ frames and for each of the simulated traffic offered load $G$ values.

Both CRA and CRDSA have degree $d=2$, i.e. each user replicates two times the layer 2 packet to be sent, and have rate $R=1$, where $R=R_{c} \cdot \log _{2}(M) . R_{c}$ is the code rate and $M$ the modulation order. The Shannon's capacity limit is assumed for the decoding threshold of the received packets as in [2], [7], [8]. For sake of completeness, we recall here the procedure for determining the correct decoding of a packet.

\footnotetext{
${ }^{7}$ It can be demonstrated exploiting the expectation operator definition and the convexity of $P_{s u c c-L 2}{ }^{i}$ for $P_{s u c c-L 2} \leq 1$ and $i \geq 0$.
}

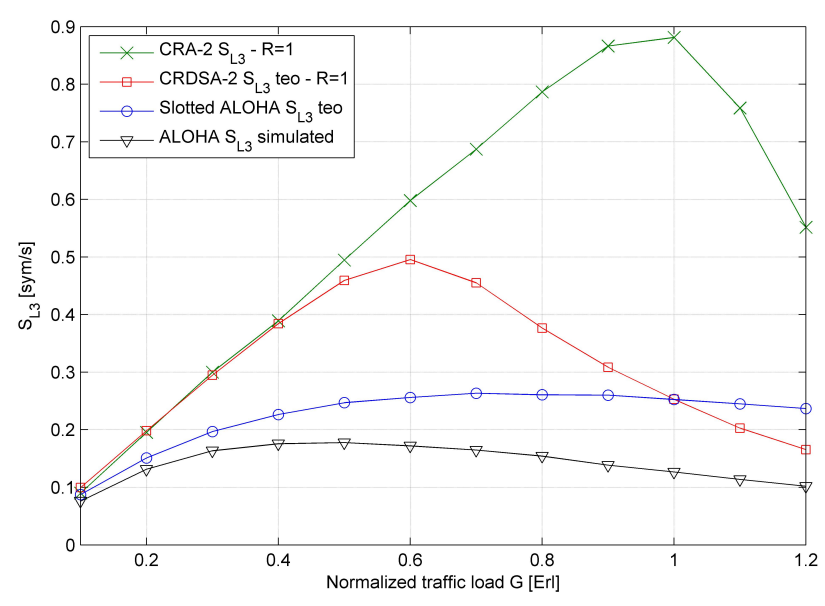

Fig. 2. Layer 3 throughput simulations with $f_{X}(x)=\frac{1}{2} \delta(x-1)+\frac{1}{2} \delta(x-2)$

We can write the SNIR decoding threshold as $S N I R_{S H A}=$ $2^{R}-1$, given the rate $R$, using the Shannon's capacity limit formula for the Additive White Gaussian Noise (AWGN) channel. We can also write the SNIR of the generic user $u$ and replica $r$ as $S N I R_{u, r}=\frac{P}{x_{u, r} \cdot P+N}$. The quantity $x_{u, r}$ is the interference ratio suffered by the packet of user $u$ and replica $r$, e.g. $x_{u, r}=1$ means that one packet is entirely colliding with the given packet. Every packet with $S N I R_{u, r} \geq S N I R_{S H A}$ is assumed to be correctly decoded.

The considered scenarios are characterized by a nominal $S N R=P / N=10 \mathrm{~dB}$ equal for each user generating traffic and the frame duration is selected to be $T_{f}=100 \mathrm{~ms}$. The layer 2 packet duration is fixed to $T_{p}=1 \mathrm{~ms}$ for the slotted schemes. Finally the SIC procedure employed in CRA and CRDSA is assumed to be ideal, i.e. every correctly decoded packet is prefectly removed from the frame as its twin replica. A maximum of $I_{\max }=10 \mathrm{SIC}$ iterations are used in the simulations.

The layer 3 throughput of the non slotted protocols, i.e. CRA and ALOHA, has been simulated, while for the slotted protocols, i.e. CRDSA and Slotted ALOHA, the throughput expression of equation (2) has been used, whereas $P_{\text {succ }-L 2}$ has been simulated.

In figure 2 the layer 3 throughput results for the case of $f_{X}(x)=\frac{1}{2} \delta(x-1)+\frac{1}{2} \delta(x-2)$ packet length distribution are shown. The green $X$ marked curve shows the CRA throughput behavior, while the red $\square$ marked curve shows the CRDSA behavior. Also the layer 3 throughput results for both Slotted ALOHA and ALOHA are shown in the figure, respectively blue $\mathrm{O}$ curve and black $\nabla$ curve.

The throughput performance is maximized by CRA for all the traffic offered load values in the simulations. The maximum layer 3 throughput reached by $\mathrm{CRA}$ is $\cong 0.88$ at $G=1$. CRDSA instead can reach a maximum layer 3 throughput of $\cong 0.49$ at $G=0.6$. Therefore, the layer 3 throughput gain looking at the maximum throughput of CRA is $88 \%$ with respect to CRDSA. Moreover, both CRA 


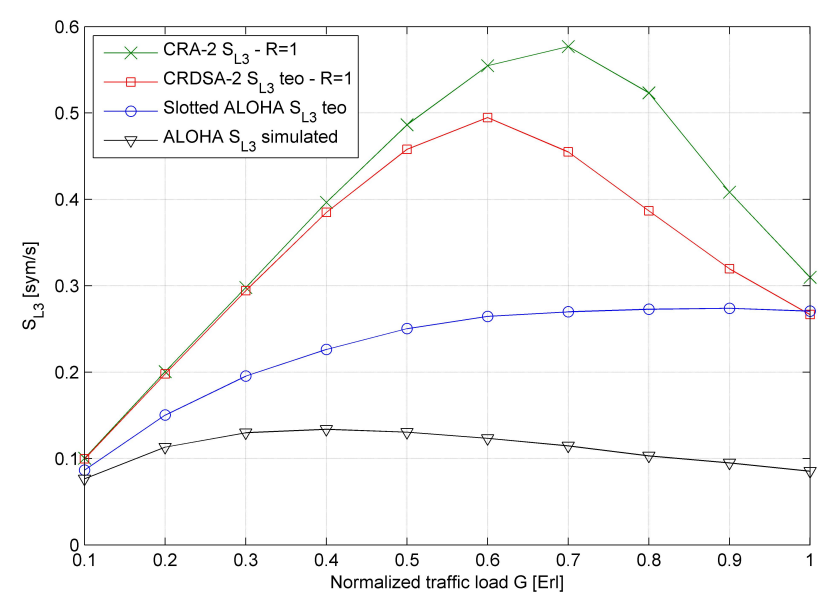

Fig. 3. Layer 3 throughput simulations with $f_{X}(x)=e^{-x}$

and CRDSA are subject to circa $10 \%$ of maximum layer 3 throughput reduction compared to the maximum layer 2 throughput, which is $\cong 0.96$ for CRA [2] and is $\cong 0.55$ for CRDSA [3]. Please note that in this case no fragmentation overhead and no padding overhead are considered for the case of slotted protocols (Slotted ALOHA and CRDSA). In this way, the results for the slotted protocols are upper bounds on the real behavior of the protocols. The maximum layer 3 throughput of Slotted ALOHA is $\cong 0.263$ at $G=0.7$, while the maximum layer 3 throughput of ALOHA is $\cong 0.178$ at $G=0.5$.

In figure 3 the layer 3 throughput results for the case of $f_{X}(x)=e^{-x}$ packet length distribution are shown. The colour and marker convention is the same as the one of figure 2 .

As in the previous case, the throughput performance is maximized by CRA for all the traffic offered load values in the simulations. The maximum layer 3 throughput reached by CRA is in this case $\cong 0.58$ at $G=0.7$. CRDSA instead can reach a similar maximum layer 3 throughput of $\cong 0.49$ at $G=0.6$. In this second case, the layer 3 throughput gain looking at the maximum throughput of CRA is $18 \%$ with respect to CRDSA. While CRA is subject to circa $48 \%$ of maximum layer 3 throughput reduction compared to the maximum layer 2 throughput (@0.96 [2]), CRDSA instead is subject to only $10 \%$ of maximum layer 3 throughput reduction

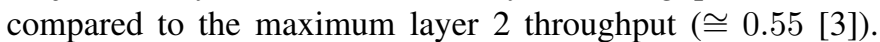
As before, no fragmentation overhead and no padding overhead are considered for the case of slotted protocols (Slotted ALOHA and CRDSA). The maximum layer 3 throughput of Slotted ALOHA is $\cong 0.274$ at $G=0.9$, while the maximum layer 3 throughput of ALOHA is $\cong 0.134$ at $G=0.4$, in line with the curves presented in [10].

In both scenarios CRA is able to outperform CRDSA in terms of layer 3 throughput for all the simulated offered load values. On the other hand, CRDSA is more robust to the different packet length distributions with respect to CRA.

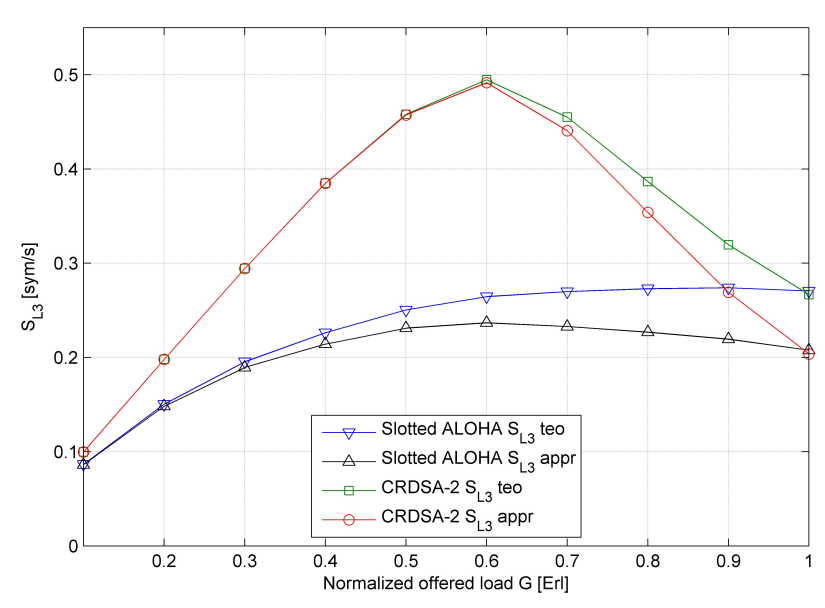

Fig. 4. Layer 3 throughput comparison between exact and approximated $P_{\text {succ }-L 3}$ and with $f_{X}(x)=e^{-x}$

\section{B. Exact and approximated layer 3 successful decoding prob- ability comparison}

In this section we show the comparison between the exact and approximated layer 3 successful decoding probability, $P_{\text {succ-L3 }}$ and $P_{\text {succ-L3-appr }}$ respectively of equations (3) and (6), and to show their impact on the layer 3 throughput.

The parameters of the simulations are the ones presented in the previous subsection and the packet length distribution considered is $f_{X}(x)=e^{-x}$.

In figure 4 this comparison is presented. The green $\square$ marked curve shows CRDSA layer 3 throughput with the exact successful decoding probability $P_{s u c c-L 3}$, while the red $\mathrm{O}$ marked curve shows the CRDSA layer 3 throughput with the approximated successful decoding probability $P_{\text {succ-L3-appr }}$. Also the layer 3 throughput results for Slotted ALOHA with the exact successful decoding probability $P_{\text {succ-L3 }}$ and with the approximated successful decoding probability $P_{\text {succ-L3-appr }}$ are depicted in the figure (blue $\nabla$ and black $\triangle$ curves respectively).

The results for CRDSA show a very good matching between the exact and approximated layer 3 throughput. In the linear region of the throughput, where $P_{\text {succ-L3 }} \cong 1$, there is no visible mismatch. Furthermore, the maximum layer 3 throughput is exactly forecast by the approximated expression. Only in the descending slope of the throughput a differences in the two curves can be found, but it can be shown that for very high levels of offered traffic load the two layer 3 throughput expressions tend to converge to the same value. In fact, it is possible to show that for $\lim _{P_{\text {succ }-L 3} \rightarrow 0} G \cdot P_{\text {succ }-L 3}=$

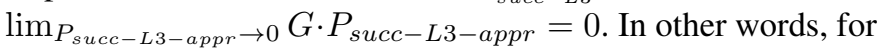
high traffic offered load values, the successful probability in both cases tends to zero. This leads to a almost zero throughput in both approximated and exact cases. The results for Slotted ALOHA show that the approximated layer 3 throughput is a lower bound of the exact layer 3 throughput and in this case it is a worse approximation than in the case of CRDSA. 
The results showed that, depending on the considered protocol, the layer 3 throughput approximation is more or less tight to the exact throughput expression. On the other hand, the approximation is a lower bound as presented in the analytical derivation of this paper (section III), therefore it can be used as a conservative starting point for protocol design.

\section{CONCLUSIONS}

The aim of the paper was to investigate the behavior of the recently presented non slotted CRA, and slotted CRDSA, RA MAC protocols in terms of layer 3 throughput, where the packets have a generic length distribution $f_{X}(x)$.

An analytical framework for the layer 3 throughput in case of slotted protocols has been derived in the paper, in order to avoid specific layer 3 simulations. In this way, the layer 3 throughput behavior can be immediately derived in closed form as soon as the successful decoding probability of the layer 2 is provided.

The results have shown that assuming FEC and with the specific parameters selected for the simulations, CRA is always able to outperform the layer 3 throughput of CRDSA. On the other hand, CRDSA showed to be more robust against variations in the form of the packet length distribution.

\section{REFERENCES}

[1] N. Abramson, "The ALOHA system: Another alternative for computer communications," in Proceedings of the 1970 Fall Joint Comput. Conf., AFIPS Conf., vol. 37, Montvale, N. J., 1970, pp. 281-285.

[2] C. Kissling, "Performance Enhancements for Asynchronous Random Access Protocols over Satellite," in Communications (ICC), 2011 IEEE International Conference on, Kyoto, Japan, June 2011, pp. 1-6.

[3] E. Casini, R. De Gaudenzi, and O. del Rio Herrero, "Contention Resolution Diversity Slotted ALOHA (CRDSA): An Enhanced Random Access Scheme for Satellite Access Packet Networks," Wireless Communications, IEEE Transactions on, vol. 6, no. 4, pp. 1408-1419, April 2007.

[4] L. G. Roberts, "ALOHA packet system with and without slots and capture," SIGCOMM Comput. Commun. Rev., vol. 5, pp. 28-42, April 1975.

[5] N. Abramson, "The Throughput of Packet Broadcasting Channels," Communications, IEEE Transactions on, vol. 25, no. 1, pp. 117-128, January 1977.

[6] G. Liva, "Graph-based analysis and optimization of contention resolution diversity slotted aloha," Communications, IEEE Transactions on, vol. 59, no. 2, pp. 477-487, february 2011.

[7] F. Clazzer and C. Kissling, "Enhanced Contention Resolution ALOHA - ECRA," in Systems, Communications and Coding (SCC), 2013 International ITG Conference on, Munich, Germany, January 2013.

[8] - "Optimum Header Positioning in Successive Interference Cancellation (SIC) based ALOHA," in Communications (ICC), 2013 IEEE International Conference on, Budapest, Hungary, June 2013, pp. 28692874.

[9] J. L. Jensen, "Sur les fonctions convexes et les inegalites entre les valeurs moyennes," Acta mathematica, vol. 30, pp. 175-193, 1905.

[10] S. Bellini and F. Borgonovo, "On the Throughput of an ALOHA Channel with Variable Length Packets," Communications, IEEE Transactions on, vol. COM-28, No. 11, pp. 1932-1935, November 1980. 